\title{
Impact of untethering of the spinal cord on urological management and antibiotic stewardship among spina bifida patients in Malaysia
}

Singh Nisheljeet

Universiti Malaya

Abu Bakar Azizi

National University of Malaysia

Kamalanathan Palaniandy

National University of Malaysia

Dharmendra Ganesan

Universiti Malaya

Teng-Aik Ong

Universiti Malaya

Azmi Alias

Hospital Kuala Lumpur

Ramalinggam Rajamanickam

National University of Malaysia

Wahib Mohammed Atroosh

Universiti Malaya

Siti Waheeda Mohd-Zin

Universiti Malaya

Andrea Lee-Shamsuddin

Universiti Malaya

Singh Nivrenjeet

Universiti Malaya

Noraishah Mydin Abdul-Aziz ( $\nabla$ noisha@ummc.edu.my )

Universiti Malaya

\section{Research Article}

Keywords: spina bifida, urinary tract infection, antibiotic stewardship, Malaysia

Posted Date: March 29th, 2022

DOI: https://doi.org/10.21203/rs.3.rs-1394466/v2 
License: (c) (i) This work is licensed under a Creative Commons Attribution 4.0 International License. Read Full License 
Impact of untethering of the spinal cord on urological management and antibiotic stewardship among spina bifida patients in Malaysia

Singh Nisheljeet ${ }^{1}$, Abu Bakar Azizi ${ }^{2}$, Kamalanathan Palaniandy ${ }^{2}$, Dharmendra Ganesan ${ }^{3}$, Teng-Aik Ong ${ }^{3}$, Azmi Alias ${ }^{4,5}$, Ramalinggam Rajamanickam ${ }^{6}$, Wahib Mohammed Atroosh ${ }^{7}$, Siti Waheeda Mohd-Zin ${ }^{1}$, Andrea Lee-Shamsuddin ${ }^{1}$, Singh Nivrenjeet ${ }^{1}$, and Noraishah Mydin Abdul-Aziz ${ }^{*}$

${ }^{1}$ Invertebrate \& Vertebrate Neurobiology Lab, Department of Parasitology, Faculty of Medicine, Universiti Malaya, 50603 Kuala Lumpur, Malaysia

${ }^{2}$ Department of Surgery, National University of Malaysia, Kuala Lumpur, Malaysia

${ }^{3}$ Department of Surgery, Faculty of Medicine, Universiti Malaya, 50603 Kuala Lumpur, Malaysia

${ }^{4}$ Department of Neurosurgery, Tunku Abdul Rahman Neuroscience Institute (IKTAR), Hospital Kuala Lumpur, 50586 Kuala Lumpur, Malaysia

${ }^{5}$ Department of Pediatric Neurosurgery, Women and Children Hospital Kuala Lumpur, 50586 Kuala Lumpur, Malaysia

${ }^{6}$ Faculty of Law, Universiti Kebangsaan Malaysia, 43600 UKM Bangi, Selangor

${ }^{7}$ Department of Parasitology, Faculty of Medicine, Universiti Malaya, 50603 Kuala Lumpur, Malaysia

*Correspondence: Noraishah Mydin Abdul-Aziz; noisha@ummc.edu.my, 


\begin{abstract}
Background

The incidence and severity of urinary tract infections (UTIs) due to spina bifida is poorly understood in Malaysia. Tethering of the spinal cord is a pathological fixation of the cord in the vertebral column that can result in neurogenic bladder dysfunction and other neurological problems. It occurs in patients with spina bifida and the authors of this study sought to determine the effects of untethering on the urological outcome of children with a tethered cord and thus identifying proper urological management for individuals with spina bifida.
\end{abstract}

\title{
Methods
}

Demographic and clinical data were collected via an online questionnaire and convenient sampling technique were used. 49 spina bifida individuals participated in this study.

\section{Results}

The diagnosis of UTIs is dependent on patients reporting cloudy and smelly urine (67\%) and urine validation $(60 \%) .18 \%$ of the respondents claimed to have been prescribed antibiotics even though they had no history of UTI. Indiscriminate prescription of antibiotics by healthcare workers further compounds the severity of UTI. Employing CISC (73\%) including stringent usage of sterile catheters (71\%) did not prevent patients from getting UTI. Overall, 33\% of our respondents reported manageable control of UTI $(0-35$ years of age $)$. All individuals below the age of $5(n=14)$ had improved urological outcome due to the untethering surgery under the guidance of the Malaysia NTD support group.

\section{Conclusion}

Spina bifida individuals may procure healthy bladder and bowel continence for the rest of their lives provided that neurosurgical and urological treatment being instigated soon after birth and continuing into adulthood.

Keyword: spina bifida; urinary tract infection; antibiotic stewardship, Malaysia 


\section{Background}

Spina bifida is a common congenital malformation that leads to lifelong complications involving the urinary tract ${ }^{1}$. Urological dysfunction is prevalent amongst patients with injuries pertaining to the spinal cord and is especially prevalent in children born with birth defects. In many countries, the major cause of death after the first year of life has been attributed to urological dysfunction which leads to kidney failure ${ }^{2,3}$. There is indefinite exposure of urological guidance in Malaysia for individuals with spina bifida from birth till the first symptom arises as urologic morbidity and mortality had become problematic for all individuals progressing into adulthood. A proper system should be in place for urological management because it is integral in ensuring that spina bifida patients have a better quality of life and improved health continuity ${ }^{4}$. Despite that, Malaysia has yet to set up a proper standard operating procedure for spina bifida individuals from birth as well as their support system towards building a better quality of life ${ }^{5,6}$.

Furthermore, kidney health accounts for approximately $70 \%$ survivability of these children $^{7}$. The use of prophylactic antibiotics is still a means of debate in the care of spina bifida individuals ${ }^{7,8}$. The health economics of Malaysians with spina bifida is not known, although a simple estimation of the Malaysian population puts the minimal expenditure Malaysia has spent thus far at day one of life at 63 million Ringgit Malaysia in a hospital setting (Ganesan, D., personal communication). In a study done on the survivability of patients of spina bifida with end-stage renal disease (ESRD), patients with spina bifida develop ESRD at younger ages than patients without spina bifida. Urological issues were the most common primary cause of $\mathrm{ESRD}^{9}$. It is not known how many Malaysians suffer irreversible kidney damage due to under supervised urological management brought upon by a birth defect. Individuals with spina bifida are at risk of progressive renal damage, in conjunction with recurrent urinary tract infections and a hostile neurogenic bladder. The impaired bladder may result in upper urinary tract deterioration, hydronephrosis, recurrent pyelonephritis and renal scarring ${ }^{10}$. Some individuals may progress to ESRD requiring dialysis. Hence, management of bladder function to prevent adverse urinary tracts infections to preserve renal function is critical.

Antibiotic usage among Malaysians with spina bifida is not known and has never been investigated. This is the first study of its kind investigating the experiences of spina bifida 
individuals and their caregivers when faced with a potential urinary tract infection. Respondents were members of a Malaysian spina bifida support group known as Malaysia Neural Tube Defects (Malaysia NTD). However, this study does not analyse the outcome of the urine culture nor does it measure in any way the patient response after appropriate antibiotic treatment.

\section{Methods}

\section{Data collection and management}

This study was carried out in accordance with the recommendation of the UMMC Medical Research Ethics Committee (MREC) with written informed consent from all respondents. Our study abided by the "Declaration of Helsinki" and informed consent was obtained from the respondents or their guardians before recruiting to the study and data collection. The study was reviewed and approved by the Medical Research and Ethics Committee (MREC (NMRR-14-1958-20549 (IIR)) of the Ministry of Health Malaysia. We advertised on social media platforms (WhatsApp and Facebook). A quantitative study aimed to spotlight the understanding of urological management among spina bifida patients within our cohort in Malaysia. A convenient sampling technique was defined; this included individuals who do ultrasound regularly, employs the usage of CISC and validated their urine sample for UTI. A total of 49 individuals responded to the questionnaire.

\section{Statistical analysis}

The data were analysed statistically using statistical packages for social sciences (SPSS), version 26. A $p$-value of 0.05 was considered significant. 


\section{Results}

In the present study, 49 respondents were reported either as spina bifida individuals (6; $12 \%)$ or the parents $(43 ; 88 \%)$ who answered the questionnaire on behalf of their spina bifida children. There were in total $53 \%$ males $(n=26)$ and $47 \%$ females $(n=23)$ spina bifida patients with a mean of 9.76 years of age. In the $0-5$ years age group, there was almost twice the number of males $(n=12)$ to females $(n=7)$. In the 6-20 years age group, there were more males $(n=11)$ compared to females $(n=8)$. Meanwhile, females were more $(n=6)$ than males $(n=1)$ in the 2135 age group (Table 1).

As many as $28(57 \%)$ individuals had their untethering surgery done, of which 22 individuals (79\%) were in the age group $<5$ years old and the remaining 6 respondents $(21 \%)$ in the age group greater than 5 years of age (Table 2$)$. The study revealed that $50 \%(n=14)$ of the respondents who are in the age group $<5$ years old, underwent untethering surgery and consequently had a significantly lower frequency of UTI, meanwhile, only $7 \%(n=2)$ in the age group $>5$ years said to have lowered their frequency of UTI after the untethering surgery ( $p=0.009)$ (Table 4). On the other hand, 12 individuals (42.9\%) reported no difference in the frequency of UTIs after the surgery. However, $18 \%(n=8)$ of the respondents reported zero occurrence of UTI which was validated by urine test and yet antibiotics were prescribed when they visited either the clinic or hospital. Furthermore, the study showed that $44.89 \%(n=22)$ of all respondents claimed to have an improved kidney function after the surgery compared to $42.86 \%(\mathrm{n}=21)$ who did not do their untethering surgery $(p=0.01)$ (Table 3$)$.

Evidently, this study has revealed that $53 \%(n=24)$ of respondents claimed to have been prescribed antibiotics during their visit to the clinic $(p=0.033)$ including 4 individuals who did not have UTI but were prescribed antibiotics regardless and $71 \%(n=32)$ of respondents claimed to have been prescribed with antibiotics during their visit to the hospital ( $p=0.003)$ including 6 individuals who did not suffer from UTI although parents and individuals with neural tube defects had been prescribed with antibiotics $(p=0.003)$ (Table 5). Besides, 67\% of respondents who visited either the clinics or hospitals were experiencing UTI symptoms such as cloudy and smelly urine, in contrast to the 13 respondents who did not exhibit these symptoms (smelly/cloudy urine) $(p=0.057)$ (Table 5). In addition, $27 \%(\mathrm{n}=12)$ and $11 \%(\mathrm{n}=5)$ of the respondents claimed to have other symptoms of UTI like stomach discomfort ( $p=0.057$ ) and back pain $(p=0.227)$ respectively. 
Our study showed that $80 \%$ of the respondents had submitted their urine sample for culture $(p=0.031)$ and a significant number of patients who gave their urine samples at the hospitals or clinics were suffering from UTI before the untethering surgery $(p=0.003$ and $p=0.033$ respectively) (Table 5). However, this study does not analyse the outcome of the urine culture nor does it measure in any way the patient response after corrects antibiotic treatment. This study also reports that $87 \%$ of respondents do kidney ultrasound regularly $(p=0.018)$ and the majority of patients goes directly to the clinic or the hospital during their first encounter with UTI ( $p=0.023$ ). Around $73 \%$ of respondents practised the use of clean intermittent selfcatheterization (CISC) as a urological management amongst spina bifida in Malaysia, meanwhile, $24 \%$ of the respondents do not employ CISC ( $p=0.514)$. In the same vein, $69 \%$ of respondents said CISC is an invasive procedure $(p=0.048)$, meanwhile, $27 \%$ of the respondents were not sure about the invasiveness of the CISC procedure. However, the degree of sterility practised is a major concern as well. $71 \%$ of the respondents used sterile catheters during every CISC procedure, whereas $29 \%$ of respondents do not employ any means of a sterile catheter $(\mathrm{p}=0.055)$.

\section{Discussion}

In Malaysia, clinics are ill-equipped to deal with complications arising from spina bifida. This is supported by the finding that the clinic-goers among spina bifida individuals in our cohort were not attending clinics specifically for the complaint of UTI. Interestingly, a significant value was obtained ( $p=0.033$ ) for handing urine samples to the clinic (Table 5). The justification from this statement comes from the fact that clinics prescribe antibiotics without knowing which antibiotic to prescribe to the spina bifida patient. Our data showed that $18 \%$ of the respondents who were given their urine for validation of UTI, were in fact ill-advisedly prescribed antibiotics for UTI, even though they had no history of suffering from urinary tract infections.

According to the Ministry of Health Malaysia, spina bifida is recognized primarily as a neurological disorder requiring the care of a neurosurgeon ${ }^{1}$ but urologists equipped to deal with a functional bladder in Malaysia is scarce. Micturition requires proper function of the bladder and urethral sphincter complex. This process requires coordination between the central and the peripheral nervous system. Disruption of this pathway caused by damage or disease can lead 
to the neurogenic bladder (NGB) with abnormal bladder storage and emptying ${ }^{2}$. Our data showed that UTI is prevalent before the untethering surgery and was found to report a significant value in improving kidney function $(p=0.01)$ and lowering the frequency of UTI after the untethering of the spinal cord $(p=0.009)$ (Table 4). The urologic representation of a patient's left kidney as seen in Figure 1, shows pre and post impact of untethering of spinal cord on kidney condition. The understanding of UTI appears when there is retention of urine in the bladder due to lack of bladder function and stimulus leading to overactivity of detrusor muscle and vesicoureteral reflux. Backflow of urine damages the kidney, builds up bacteria due to prolonged urine retention which will cause infection and fever. Untethering of the spinal cord as seen in Figure 2, helps to release the pressure of the tethered nerves, rejuvenates and stimulates the bladder for better voiding ${ }^{14}$. UTI in patients with neurogenic bladder remains a challenge for urologists and other clinicians. This is because altered anatomy and bladder mechanics, frequent catheterization, impaired sensation, and immunologic dysfunction make patients with neurogenic bladder uniquely prone to infections and difficult to diagnose and treated appropriately. Although advances in medical care have significantly reduced the morbidity and mortality of urinary tract infection (UTI) in patients with neurogenic bladder (NGB), ill-advised use of antibiotics showed $10 \%$ to $15 \%$ of patients with NGB die from sepsis of urinary origin ${ }^{15}$. However, having the correct urological surgical intervention can prevent infections and lead to a better quality of life as well as ensuring a lifetime with a healthy pair of kidneys.

According to our study, Malaysians probably do not know how to do CISC properly, although the use of sterile catheters shows a better prognosis than re-using catheters. There should not be any problem for spina bifida patients to have healthy, functioning kidneys their whole life but deterioration of kidneys is often seen on spina bifida patients and this is because patients do not do subsequent follow-ups with their urologist. That is to say, if they even have one seeing as there are very few skilled urologists in Malaysia, most spina bifida patients will be referred to a nephrologist instead. The question most asked by parents of babies with spina bifida to their urology consultant is whether they need to employ CISC in the management of their child's urological needs. The second question is when do they need to begin and the third question asked is how often does CISC need to be done. In most clinical settings, the question of whether there could be other means apart from CISC more well suited for the spina bifida child is often not asked nor investigated. A study done on family compliance towards CISC 
shows that although most parents dislike CISC, they complied with the suggested management ${ }^{16}$.

Furthermore, a study on the association between UTI and CISC showed that infants and toddlers with spina bifida who were initially managed with spontaneous voiding had a lower risk of UTI than those managed with CISC and this is evidently shown in our study by employing the usage of CISC to reduce the recurrence of UTI but the association is not statistically significant $(p=0.514)$. However, patients who do CISC after a period of voiding did not have a significantly different risk of UTI which suggest that early initiation of CISC may not be warranted in all infants with spina bifida ${ }^{17}$. In fact, CISC at an early age may potentially have a detrimental effect on the child like trauma, loss of sensory control and reflexes ${ }^{17}$. CISC (Lapides et al., 1972), which has been a foundation used by most urologists and other clinicians as a modality for urological management amongst spina bifida individuals with neurogenic or atonic bladders encompassing urinary retention, incontinence and infection needs to be re-assessed. The Lapides technique (1972) need not necessarily be the gold standard with the advent of science and technology. The quality of life of spina bifida children was improved as our finding suggests that $100 \%(\mathrm{n}=14)$ of respondents in the age group $0-5$ years old had lowered their UTI frequency after the untethering of the spinal cord (Table 3) especially if performed at an early age ${ }^{18}$. This is further strengthened as our data showed that $45 \%$ of the respondents had improved their kidney function after the untethering surgery $(p=0.01)$. Moreover, families who do not wish to spend extra on catheters and advice from clinics or hospitals especially outside of Kuala Lumpur appears to be focused on budget constraints. Age and social media networking improve the level of experience and understanding of urological management ${ }^{19,20}$.

Respondents who took antibiotics during their visit to the clinic or hospital were in fact suffering urinary tract infection (UTI) (Table 5) whereby $82 \%$ of the respondents attended clinic/ hospital when they first suspected UTI ( $p=0.023$ ). This suggests that individuals with UTIs in Malaysia are able to manage and mobilise themselves to get the correct treatment. On average there was an increase of 500g of urine upon untethering of spinal cord and frequency of diapers changed increased as well (personal communication with the respondents). Before the untethering surgery, the frequency of diaper changed occurs every 4 hours which accumulates to a total of 6 diapers changed in a day. In comparison to after the untethering 
surgery, on average, the diapers were changed every 2 hours which meant doubling the total number of diaper change.

\section{Conclusions}

Asymptomatic bacteriuria should not be treated in most patients, but, given the absence of a standardized definition of UTI in this patient population, efforts to optimally diagnose, treat, and study recurrent UTI in patients with neurogenic bladder have been significantly hampered. This area requires a major effort in future studies. Antibiotic stewardship in Malaysia is considered almost completely absent based upon this pilot study as none of the respondents reported having to address the issue of urine culture analysis. Therefore, the prevention of UTIs in this scenario is considered critical. We recommend hospitals that process urine for culture should set the standards for spina bifida patient care as well as pursuing a larger dataset that would provide a more convincing prevalence association between untethering the spinal cord at an early age and a better prognosis on the urological outcome. 


\section{Declarations}

\section{Ethics approval and consent to participate}

- This study was carried out in accordance with the recommendation and approval of the Medical Research and Ethics Committee (MREC) of Ministry of Health Malaysia (Reference number: NMRR-14-1958-20549 (IIR)).

- Informed consents were obtained from the respondents or their guardians.

\section{Consent for publication}

- None of the contributing authors have any conflict of interest, including specific financial interests or relationships and affiliations relevant to the subject matter or materials discussed in the manuscript.

\section{Availability of data and materials}

- The datasets used and/or analysed during the current study are available from the corresponding author on reasonable request.

\section{Competing interests}

- None of the contributing authors have any conflict of interest, including specific financial interests or relationships and affiliations relevant to the subject matter or materials discussed in the manuscript.

\section{Funding}

- Supported by Fundamental Research Grant Scheme FRGS/1/2019/SKK08/UM/02/17 (FP090-2019A) and FRGS/1/2019/SKK06/UKM/02/06 from the Ministry of Education Malaysia to N.M.A-A. and A.B.A. The authors express their appreciation to the investigators and patients who participated in this study.

Author Contributions: NMA-A, WMA, T-AO and SN1 conceived and designed the study. NMA-A, WMA, SWM-Z, SN1, AL-S and SN2 performed the study (SN1 is Singh Nisheljeet; SN2 is Singh Nivrenjeet). NMAA, WMA, ABA, DG, AA, KP, and T-AO analysed the data. NMA-A, WMA, AL-S. and SN1 drafted the manuscript. All authors contributed to manuscript revision, proofread and approved the submitted version. 


\section{References}

1. McKibben, M. J., Seed, P., Ross, S. S., \& Borawski, K. M. (2015). Urinary tract infection and neurogenic bladder. Urologic Clinics of North America, 42(4), 527-536. https://doi.org/10.1016/j.ucl.2015.05.006

2. Ahmad, I., \& Granitsiotis, P. (2007). Urological follow-up of adult spina bifida patients. Neurourology and Urodynamics, 26(7), 978-980. https://doi.org/10.1002/nau.20447

3. Szymanski, K. M., Misseri, R., Whittam, B., Adams, C. M., Kirkegaard, J., King, S., Kaefer, M., Rink, R. C., \& Cain, M.P. (2015). Mortality after bladder augmentation in children with spina bifida. The Journal of Urology, 193(2), 643-648.

4. SPA: Kategori Orang Kurang Upaya (OKU). c2021. Suruhanjaya Perkhidmatan Awam Malaysia; [accessed 2021 August 28]. https://www.spa.gov.my/spa/panduan/kategoriorang-kurang-upaya-oku

5. Ong LC, Norshireen NA, Chandran V. Maternal mental health in families of children with spina bifida. World J Pediatr. 2011 Feb;7(1):54-9. doi: 10.1007/s12519-011-0246z. Epub 2010 Dec 30. PMID: 21191777

6. Kanaheswari, Y., Razak, N. N., Chandran, V., \& Ong, L. C. (2011). Predictors of parenting stress in mothers of children with spina bifida. Spinal Cord, 49(3), 376-380.

7. Filler G, Gharib M, Casier S, Lodige P, Ehrich JH, Dave S. Prevention of chronic kidney disease in spina bifida. Int Urol Nephrol 2012; 44: 817-27. doi: 10.1007/s11255010-9894-5.

8. Zegers, S. H., Dieleman, J., van der Bruggen, T., Kimpen, J., \& de Jong-de Vos van Steenwijk, C. (2017). The influence of antibiotic prophylaxis on bacterial resistance in urinary tract infections in children with spina bifida. BMC Infectious Diseases, 17(1), 63. 
9. Ouyang, L., Bolen, J., Valdez, R., Joseph, D., Baum, M. A., \& Thibadeau, J. (2015). Characteristics and survival of patients with end stage renal disease and spina bifida in the United States renal data system. The Journal of Urology, 193(2), 558-564.

10. Joseph, D. B., Baum, M. A., Tanaka, S. T., Frimberger, D. C., Misseri, R., Khavari, R. \& Wood, H. (2020). Urologic guidelines for the care and management of people with spina bifida. Journal of pediatric rehabilitation medicine, (Preprint), 1-10.

11. Charan, J., \& Biswas, T. (2013). How to calculate sample size for different study designs in medical research? Indian Journal of Psychological Medicine, 35(2), 121.

12. Schlesselman JJ. 1974. Sample size requirements in cohort and case-control studies of disease. Am J Epidemiol. 99(6):381-384.

13. Sahmat, A., Gunasekaran, R., Mohd-Zin, S. W., Balachandran, L., Thong, M. K., Engkasan, J. P., \& Abdul-Aziz, N. M. (2017). The prevalence and distribution of spina bifida in a single major referral center in Malaysia. Frontiers in Pediatrics, 5, 237

14. Hsieh, M. H., Perry, V., Gupta, N., Pearson, C., \& Nguyen, H. T. (2006). The effects of detethering on the urodynamics profile in children with a tethered cord. Journal of Neurosurgery: Pediatrics, 105(5), 391-395.

15. Leoni, M. G., \& De Ruz, A. E. (2003). Management of urinary tract infection in patients with spinal cord injuries. Clinical Microbiology and Infection, 9(8), 780-785.

16. Borzyskowski, M., Cox, A., Edwards, M., \& Owen, A. (2004). Neuropathic bladder and intermittent catheterization: social and psychological impact on families. Developmental Medicine and Child Neurology, 46(3), 160-167. https://doi.org/10.1017/S0012162204000295

17. Kaye, I. Y., Payan, M., \& Vemulakonda, V. M. (2016). Association between clean intermittent catheterization and urinary tract infection in infants and toddlers with spina bifida. Journal of Pediatric Urology, 12(5), 284.e1-284.e6. https://doi.org/10.1016/j.jpurol.2016.02.010

18. Tseng, J. H., Kuo, M. F., Kwang Tu, Y., \& Tseng, M. Y. (2008). Outcome of untethering for symptomatic spina bifida occulta with lumbosacral spinal cord tethering in 31 patients: analysis of preoperative prognostic factors. The Spine Journal: official journal of the North American Spine Society, 8(4), 630-638.

19. Tomas, C. C., Oliveira, E., Sousa, D., Uba-Chupel, M., Furtado, G., Rocha, C., Teixeira, A., Ferreira, P., Alves, C., Gisin, S., Catarino, E., Carvalho, N., Coucelo, T., Bonfim, L., Silva, C., Franco, D., Gonzalez, J. A., Jardim, H. G., Silva, R., Baixinho, C. L., Rama, L. (2016). Proceedings of the 3rd IPLeiria's International Health Congress: 
Leiria, Portugal. 6-7 May 2016. BMC Health Services Research, 16 Suppl 3(Suppl 3), 200.

20. Ellis, J., Vassilev, I., Kennedy, A., Moore, M., \& Rogers, A. (2019). Help seeking for antibiotics; is the influence of a personal social network relevant? BMC Family Practice, 20(1), 63. https://doi.org/10.1186/s12875-019-0955-2

21. Kanaheswari, Y. \& Mohd Rizal, A.M. (2015). Renal scarring and chronic kidney disease in children with spina bifida in a multidisciplinary Malaysian centre. Journal of Paediatrics and Child Health, 51(12), 1175-1181.

22. Prieto, J., Murphy, C. L., Moore, K. N., \& Fader, M. (2014). Intermittent catheterisation for long-term bladder management. The Cochrane Database of Systematic Reviews, (9), CD006008.

23. Madero-Morales, P. A., Robles-Torres, J. I., Vizcarra-Mata, G., Guillén-Lozoya, A. H., Mendoza-Olazarán, S., Garza-González, E., \& Gutiérrez-González, A. (2019). Randomized clinical trial using sterile single use and reused polyvinylchloride catheters for intermittent catheterization with a clean technique in spina bifida cases: short-term urinary tract infection outcomes. The Journal of Urology, 202(1), 153-158. https://doi.org/10.1097/JU.0000000000000244

24. Kiddoo, D., Sawatzky, B., Bascu, C. D., Dharamsi, N., Afshar, K., \& Moore, K. N. (2015). Randomized crossover trial of single use hydrophilic coated vs multiple use polyvinylchloride catheters for intermittent catheterization to determine incidence of urinary infection. The Journal of Urology, 194(1), 174-179. https://doi.org/10.1016/j.juro.2014.12.096

25. Faleiros, F., Warschausky, S., Käppler, C., Schutt, W., Cintra, M.M., Rabeh, S.A., \& Gimenes, F. R. (2019). Bladder self-management in the transition to adulthood with spina bifida in 3 countries: a comparative study. Journal of Wound Ostomy \& Continence Nursing, 46(4), 321-326. doi: 10.1097/WON.0000000000000545

26. Lapides, J., Diokno, A. C., Silber, S. J., \& Lowe, B. S. (1972). Clean, intermittent selfcatheterization in the treatment of urinary tract disease. The Journal of Urology, 107(3), 458-461. 


\section{Figures}

Figure 1: Kidney ultrasound of pre and post tethered cord release. A) Normal left kidney after the untethering surgery; B) Dilated left kidney before untethering surgery

Figure 2: MRI scan of a spina bifida patient at Lumbar region. (A) Tethered cord at Lumbar 1 and Lumbar 2 (L1/L2) before untethering surgery (B) Post untethering surgery shows release of tethered cord

\section{Tables}

Table 1: General characteristics of respondents $(n=49)$

Table 2: Association of the age of untethering surgery with the impact on the frequency of UTI $(\mathrm{n}=28)$

Table 3: Impact of completing the untethering surgery on the improvement of kidney function $(n=49)$

Table 4: Impact of completing the untethering surgery on the frequency of UTI $(n=49)$

Table 5: Association of different risk factors with the UTI before the untethering surgery $(n=45)$

\section{Supplementary material}

1. Additional file 1

Title: Survey on Urological Management among Spina Bifida individuals

Description of Data: Questionnaires pertaining to urological management among spina bifida individuals in order to assess the level of understanding of the related disease. 
Figures

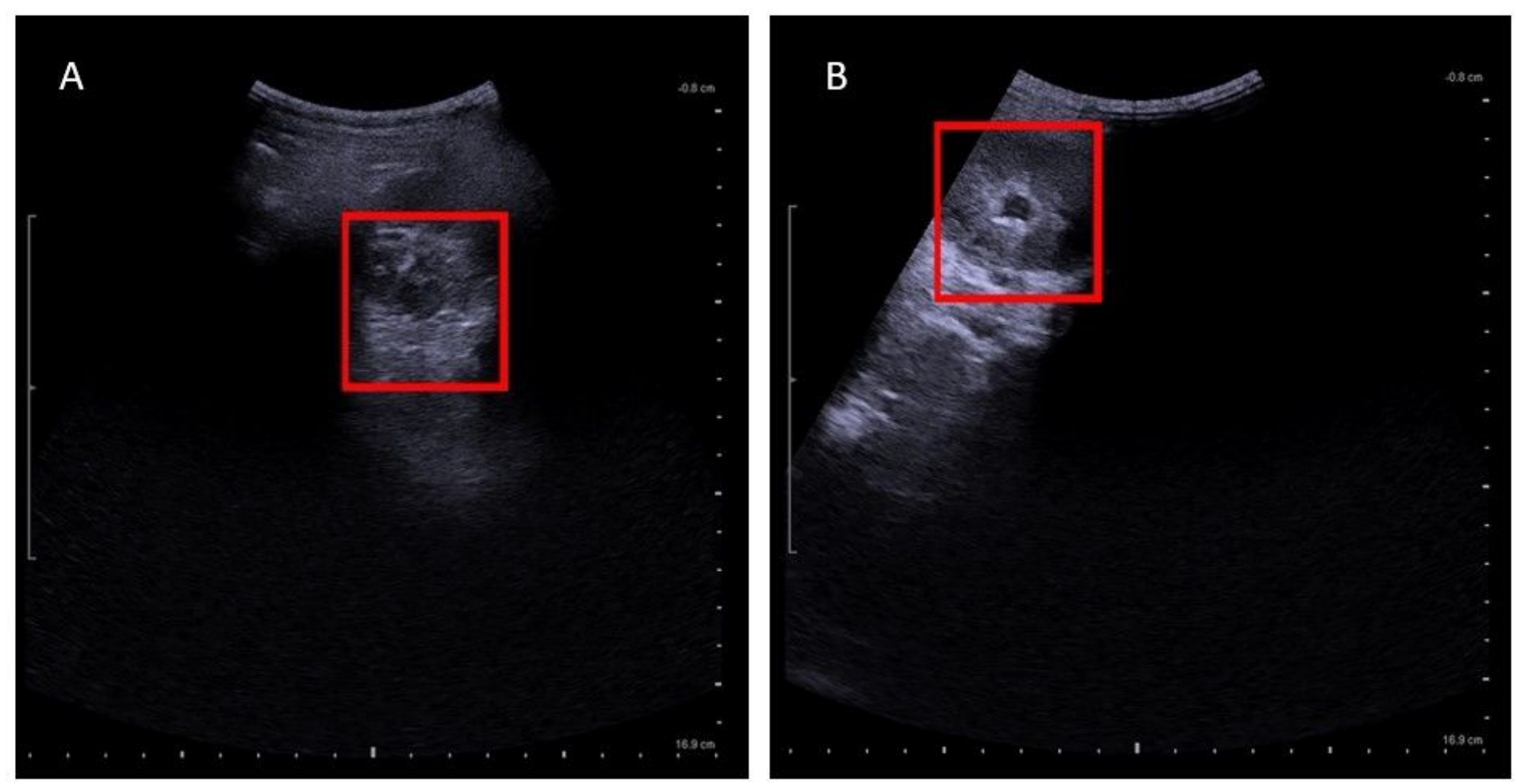

Figure 1

Kidney ultrasound of pre and post tethered cord release. A) Normal left kidney after the untethering surgery; B) Dilated left kidney before untethering surgery
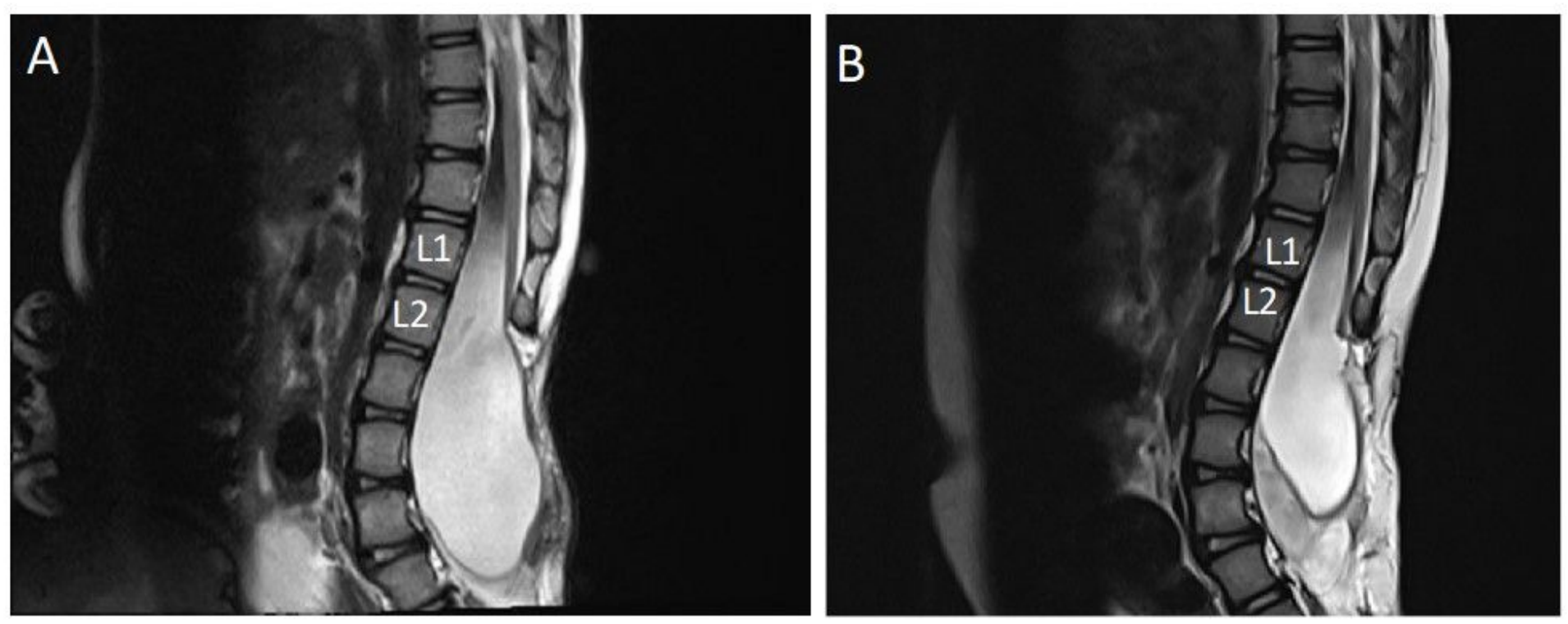

Figure 2 
MRI scan of a spina bifida patient at Lumbar region. (A) Tethered cord at Lumbar 1 and Lumbar 2 (L1/L2) before untethering surgery (B) Post untethering surgery shows release of tethered cord

\section{Supplementary Files}

This is a list of supplementary files associated with this preprint. Click to download.

- AdditionalFile1UrologyQuestionnaires.pdf 